\title{
Associations of Estrogen Receptor, Progesterone Receptor, Human Epidemic Growth Factor Receptor-2 and Ki-67 with Ultrasound Signs and Prognosis of Breast Cancer Patients
}

\author{
Xingjuan Zhao' \\ Xuan Yang' \\ Lei $\mathrm{Fu}^{2}$ \\ Keda $\mathrm{Yu}^{3}$ \\ 'Department of Mammary Gland, Shanxi \\ People's Hospital, Taiyuan City, Shanxi \\ Province, 030000, People's Republic of \\ China; ${ }^{2}$ Department of Surgery, Shanxi \\ Provincial General Team Hospital of the \\ Chinese People's Armed Police Force, \\ Taiyuan City, Shanxi Province, 030000, \\ People's Republic of China; ${ }^{3}$ Department \\ of Mammary Gland, Fudan University \\ Cancer Hospital, Taiyuan City, Shanxi \\ Province, 030000, People's Republic of \\ China
}

\begin{abstract}
Objective: The functions of estrogen receptor (ER), progesterone receptor (PR), human epidermal growth factor receptor 2 (HER2) and Ki-67 in breast cancer have been explored. This study was carried out to explore ER, PR, HER-2 and Ki-67 expression levels in breast cancer patients and their relationship with ultrasound signs and prognosis.

Patients and Methods: A total of 274 female primary breast cancer patients received preoperative ultrasound examination. ER, PR, HER-2 and Ki-67 expression levels in breast cancer tissues were detected by immunohistochemical staining after surgery. The correlations of ER, PR, HER-2 and Ki-67 expression with ultrasound signs and prognosis of breast cancer patients were analyzed.

Results: The positive expression rate of ER, PR and HER-2 and Ki-67 high expression in 274 breast cancer patients was $73.36 \%$ (201/274), 59.85\% (164/274), 24.09\% (66/274) and $66.06 \%$ (181/274), respectively. ER-positive expression had association with lymph node metastasis (LNM) and blood flow grading; HER-2-positive expression was associated with LNM, while Ki-67-positive expression was related to the tumor diameter, LNM, and blood flow grading. LNM and Ki-67 high expression were risk factors for OS; PR-positive was a protective factor for OS; TNM stage, tumor diameter, LNM and Ki-67 high expression were risk factors for DFS in breast cancer patients.

Conclusion: ER, PR, HER-2 and Ki-67 in breast cancer are related to the ultrasound signs and prognosis of breast cancer patients. The joint detection of multiple indicators provides a reference for the individualized treatment of targeted drugs.
\end{abstract}

Keywords: estrogen receptor, progesterone receptor, human epidemic growth factor receptor-2, Ki-67, breast cancer, ultrasound signs, prognosis

\section{Introduction}

Breast cancer is a complicated disease that has the characteristics of molecular and genetic diversity, contributing to the identification of several fundamentally varying subtypes. ${ }^{1}$ The morbidity of breast cancer has been increasingly elevated and is now regarded a commonly seen cancer among Chinese women. ${ }^{2}$ Breast cancer initiation and progression are caused by different endogenous or exogenous reasons, including genetics, endocrine level, environment as well as lifestyle/habitat. ${ }^{3}$ Evidence has shown that breast lumps, skin changes, enlarged axillary lymph nodes, non-gestational nipple discharge as well as nipple and/or areola
Correspondence: Xingjuan Zhao Department of Mammary Gland, Shanxi People's Hospital, No. 29 Shuangtasi Street, Taiyuan City, Shanxi Province, 030000, People's Republic of China Email zhaoxingjuan2020@I63.com 
abnormalities are the main clinical symptoms of breast cancer. ${ }^{4}$ Surgery, chemotherapy, immunotherapy, endocrine therapy, and targeted therapy are the comprehensive treatments for breast cancer. ${ }^{5}$ However, breast cancer survivors are still confronted with risks of locoregional recurrence irrespective of different treatments, ${ }^{6}$ which make the recognition of effective biomarkers in breast cancer diagnosis more important.

Nowadays, the molecular diagnostic technique has drawn some research interests due to its benefit in the diagnostic and prognostic assessment of breast cancer. ${ }^{7}$ Breast cancer is traditionally assorted in the light of the presence of the steroid hormones, the receptors (estrogen receptor (ER), progesterone receptor (PR), human epidemic growth factor receptor-2 (HER2), as well as proliferation index Ki-67. ${ }^{5}$ Currently, the immunohistochemistry-based molecular classification is suggested to have a close relationship with breast cancer prognosis, which is considered as a standard regimen for selecting the most useful approaches in breast cancer patient. ${ }^{8}$ For breast cancer, the ER and PR are the initial predictive molecular biomarkers. Breast cancer patients who have positive ER and PR generally possess high sensitivity to hormone therapy. ${ }^{9}$ HER2 gene is an oncogene in epidermal growth factor receptor (EGFR) family, and HER2 expression abnormality has close correlation with the onset, development, invasion, as well as metastasis of breast cancer. ${ }^{10,11} \mathrm{Ki}-67$ is known as a nuclear protein belonging to tumor proliferationassociated gene to embody cell proliferation, which closely correlates to the differentiation and tumor metastasis of breast cancer. ${ }^{12}$ In view of these aforementioned references, we unearthed this current research to explore ER, PR, HER2 and Ki-67 expression levels with ultrasound signs and prognosis in breast cancer patients.

\section{Patients and Methods}

\section{Ethical Approval}

Specimen collection in our experiment was approved by the ethics committee of Shanxi People's Hospital. All patients provided the informed consents. The study followed the Declaration of Helsinki.

\section{Study Subjects}

Two hundred and seventy-four cases of breast cancer patients who had been treated for the first time in three third-class hospitals in our city from June 2017 to May 2019 were included in this study. The paraffin tissue samples of all study subjects were collected for the detection of ER, PR, HER-2 and Ki-67 expression levels in breast cancer. Patients who were diagnosed with breast cancer by ultrasound and histopathological examination and who met the relevant diagnostic criteria in the National Comprehensive Cancer Network (NCCN) Guidelines Breast Version 2.2013 ${ }^{13}$ were enrolled into our study. Patients were excluded if they conformed to the exclusion criteria: those with pregnancy, menstrual cycle, moderate to severe anemia, or unexplained vaginal bleeding; those with other malignant tumors; those with serious diseases of important organs (eg, heart, liver, and kidney); and those with incomplete clinical data. All 274 patients were followed up, and the follow-up rate was $100 \%$. The follow-up deadline was May 2020 and the follow-up time lasted 6-36 months. The patient's overall survival (OS, the interval from the date of surgery to death due to any cause) and disease-free survival (DFS), the time interval from the date of surgery to the first cancer recurrence, distant metastasis, death, and second primary tumor) were recorded.

\section{Ultrasonic Examination}

The ultrasonic examination for all patients was performed by Color Doppler ultrasound diagnostic instrument (660SSA-660A, Toshiba Tungaloy Co Ltd, Kawasaki, Japan) and iU-Elite 550A (Philips, Netherlands), with probe frequencies of $4-10 \mathrm{MHz}$ and $7.5-12 \mathrm{MHz}$. During the examination, the patients were in supine, their arms were raised naturally, and both sides of the breast and armpit were fully exposed. The location, size, shape, margin, microcalcification, and axillary lymph node enlargement of the lesion were firstly scanned, and then the blood flow inside and around the lesion were examined by the color Doppler flow imaging. Two senior radiographers independently read the film in a blind manner, recorded the lesion size, spicule signs at the edges, the blood flow grading, and the presence or absence of microcalcification and lymph node metastasis (LNM).

\section{Immunohistochemical Staining}

Breast cancer tissues were fixed for 24 hours with $10 \%$ formaldehyde, routinely dehydrated, cleared, embedded in paraffin, and sliced into $5 \mu \mathrm{m}$ continuous sections. The slices were baked at $65^{\circ} \mathrm{C}$, dewaxed with xylene, hydrated with gradient ethanol, added dropwise with $3 \%$ $\mathrm{H}_{2} \mathrm{O}_{2}$ and placed in a $37^{\circ} \mathrm{C}$ incubator for 10 minutes to inactivate endogenous peroxidase. After microwave 
heating for antigen repair and normal goat serum blocking, the slices were cultured at $4{ }^{\circ} \mathrm{C}$ with primary antibody ER, PR, HER-2 and Ki-67 overnight, and then subjected to biotin-labeled secondary antibody incubation at room temperature for 30 minutes the next day. After that, the slices were developed by diaminobenzidine, counterstained by hematoxylin, followed by differentiation with hydrochloric acid ethanol, dehydration with gradient ethanol, clearing with xylene, and blocking with neutral gum, and then captured under a microscope. Phosphatebuffered saline replaced the primary antibody for a negative control. All antibodies were purchased from Zhongshan GoldenBridge (Beijing, China). The positive criterion for ER and PR is more than $1 \%$ of tumor cell nuclear staining. ${ }^{14}$ According to the criteria provided by Dako (Glostrup), HER-2 status is classified as $0,1+, 2+$, or $3+$, and tumor scores of 0 and $1+$ are considered to be negative. ${ }^{15} \mathrm{Ki}-67$ marker index (14\% or more tumor cells with nuclear staining) is considered to be highly expressed. ${ }^{16}$

\section{Statistical Analysis}

SPSS 21.0 was utilized for data analysis. The enumeration data was expressed as a percentage, and the $\chi^{2}$ test was used for analysis. Correlation was analyzed by Spearman rank correlation analysis; Kaplan-Meier analysis and COX multivariate regression analysis were implemented for determining the influencing factors for the prognosis of breast cancer patients. $P<0.05$ meant the statistical difference.

\section{Results}

\section{General Data and Analysis of Ultrasound Features of Breast Cancer Patients}

The average age of 274 breast cancer patients was $(47.1 \pm 8.5)$ years, of which $58.39 \%(160 / 274)$ aged below 50 years, and $13.14 \%(36 / 274)$ had a family history of this disease. As for TNM stage, $71.90 \%$ $(197 / 274)$ and $28.10 \%(77 / 27)$ were in stages I-II and III-IV, respectively. Others such as menopausal status, histological type and degree of differentiation are shown in Table 1. Ultrasound examination results showed that among the 274 patients with breast cancer, 123 cases had tumor diameter less than $2 \mathrm{~cm}, 151$ cases had tumor diameter equal to or more than $2 \mathrm{~cm}$; 101 cases, 129 cases, and 111 cases had LNM, spicule signs, and calcification at the edge of the
Table I General Data and Analysis of Ultrasound Features of Breast Cancer Patients

\begin{tabular}{|c|c|c|c|}
\hline \multicolumn{2}{|l|}{ Item } & \multirow{3}{*}{$\begin{array}{l}\mathbf{n} \\
75 \\
52\end{array}$} & \multirow{3}{*}{$\begin{array}{l}\text { Percentage } \\
59.06 \\
40.94\end{array}$} \\
\hline Age (years) & $<50$ & & \\
\hline & $\geq 50$ & & \\
\hline Family history of & Yes & 9 & 7.09 \\
\hline breast cancer & No & 118 & 92.91 \\
\hline \multirow[t]{2}{*}{ Menopausal status } & Premenopause & 69 & 54.33 \\
\hline & Postmenopause & 58 & 45.67 \\
\hline \multirow[t]{2}{*}{ TNM stage } & I-II & 102 & 80.31 \\
\hline & III-IV & 25 & 19.69 \\
\hline \multirow[t]{3}{*}{ Histological type } & $\begin{array}{l}\text { Infiltrating ductal } \\
\text { carcinoma }\end{array}$ & 121 & 95.27 \\
\hline & Infiltrating lobular & 5 & 3.94 \\
\hline & $\begin{array}{l}\text { carcinoma } \\
\text { Others }\end{array}$ & I & 0.79 \\
\hline \multirow{3}{*}{$\begin{array}{l}\text { Degree of } \\
\text { differentiation }\end{array}$} & High & 34 & 26.77 \\
\hline & Moderate & 66 & 51.97 \\
\hline & Poor & 27 & 21.26 \\
\hline \multirow[t]{2}{*}{ Tumor diameter $(\mathrm{cm})$} & $<2$ & 51 & 40.16 \\
\hline & $\geq 2$ & 76 & 59.84 \\
\hline Lymph node & Yes & 39 & 30.71 \\
\hline metastasis & No & 88 & 62.29 \\
\hline \multirow[t]{2}{*}{ Spicule sign } & Yes & 56 & 44.09 \\
\hline & No & 71 & 55.91 \\
\hline \multirow[t]{2}{*}{ Calcifications } & Yes & 47 & 37.00 \\
\hline & No & 80 & 62.99 \\
\hline \multirow[t]{2}{*}{ Blood flow grading } & $0-1$ & 44 & 34.65 \\
\hline & $2-3$ & 83 & 65.35 \\
\hline
\end{tabular}

lesion. In the blood flow grading, grades $0-1$ and grades 2-3 were 105 cases and 169 cases, respectively (Table 1).

\section{Expression and Correlation Analysis of ER, PR, HER-2 and Ki-67 in Patients with Breast Cancer}

In breast cancer patients, the positive rates of ER, PR and HER-2 were $73.36 \%$ (201/274), 59.85\% (164/274), and $24.09 \%$ (66/274); and high expression rate of Ki-67 was $66.06 \%(181 / 274)$, the location of each immunohistochemical positive expression is shown in Figure 1. The results of Spearman correlation analysis showed (Table 2) that ER has a positive relation with PR expression $(\mathrm{r}=0.50, P<$ 0.001); ER had a negative relationship with HER-2 and 
A

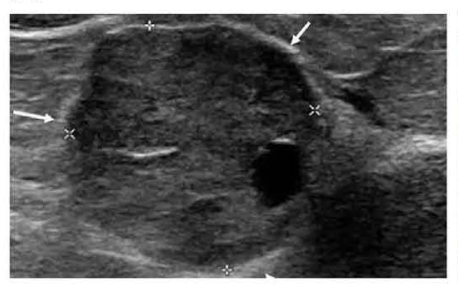

C

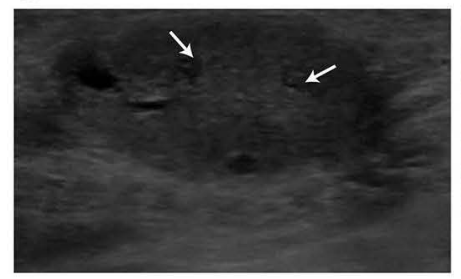

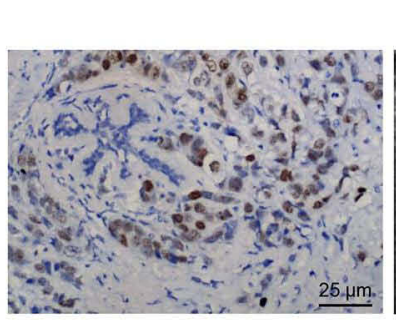

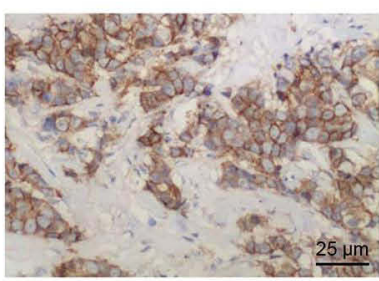

B
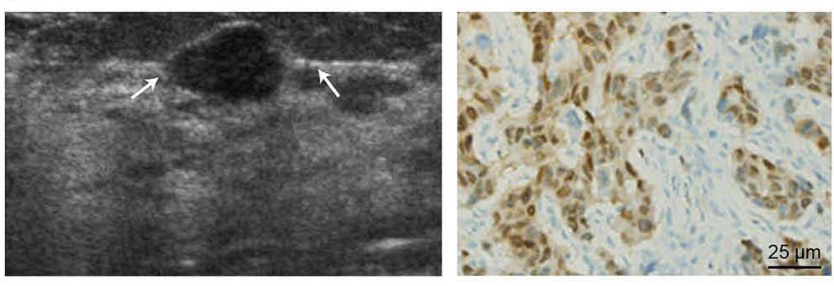

D
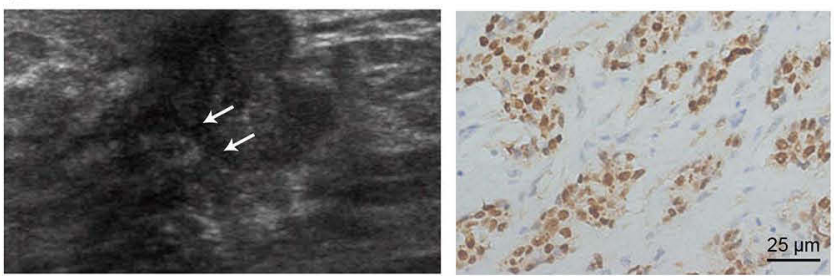

Figure I The ultrasonographic image and ER, PR, HER-2 and Ki-67 expression in breast cancer patients. (A) Burr-like edges of breast cancer mass (showed by white arrows), and ER-positive rate in breast cancer patients by immunohistochemical staining (400x). (B) Rich blood flow signal of breast cancer mass (showed by white arrows), and PR-positive rate in breast cancer patients immunohistochemical staining (400x). (C) Lymph node metastasis of breast cancer ultrasound imaging (showed by white arrows), and HER-2-positive rate in breast cancer patients immunohistochemical staining (400x). (D) Small calcifications of breast cancer mass (showed by white arrows), and $\mathrm{Ki}-67$ high expression in breast cancer patients immunohistochemical staining $(400 \times)$.

Ki-67 expression $(r=-0.53 ; r=-0.43$, both $P<0.001)$; PR was negatively related to HER-2 and Ki-67 expression ( $r=-0.36 ; r=-0.57$, both $P<0.001)$; there was a positive association between HER-2 and Ki-67 expression ( $r=$ $0.40, P<0.001)$.

\section{Relationship Between ER, PR, HER-2 and Ki-67 Expression Levels and the Characteristics of Ultrasound Signs in Breast Cancer Patients}

Breast patients with ER-positive expression had high proportion of the absence of LNM and blood flow grades $0-1$; those with HER-2-positive expression had high proportion of presence of LNM; those with Ki-67 high expression had high proportion of tumor diameter equal to or more than $2 \mathrm{~cm}$, presence of LNM and blood flow grades 2-3 (all $P<$ 0.05 ; Table 3).
Relationship Between ER, PR, HER-2 and Ki-67 Expression Levels and Prognosis in

\section{Breast Cancer Patients}

Among 274 breast cancer patients, the OS rate was $86.13 \%$ (236/274), and the DFS rate was $79.99 \%(219 /$ 274). Among these patients, PR protein-positive and Ki-67 high expression had a correlation with OS and DFS (all $P<0.05$ ), while no association was found between ER with DFS, or HER-2 protein-positive with OS and DFS (both $P>0.05$ ) (Figure 2). Furthermore, COX multivariate regression analysis was implemented to analyze the elements affecting OS and DFS in patients with breast cancer. All the above elements were enrolled in the COX multivariate regression and LR method was used for analysis. The results showed that LNM and Ki-67 high expression were the risk factors for OS, and PR positive was the protective factor of OS; and TNM stage, tumor diameter,

Table 2 Expression and Correlation Analysis of ER, PR, HER-2 and Ki67 in Breast Cancer Patients

\begin{tabular}{|l|l|l|l|l|l|l|l|l|}
\hline & \multicolumn{2}{l}{ ER } & \multicolumn{2}{l}{ PR } & \multicolumn{2}{l|}{ HER-2 } & \multicolumn{2}{l|}{ Ki67 } \\
\cline { 2 - 10 } & $\boldsymbol{r}$ & $\boldsymbol{P}$ & $\boldsymbol{r}$ & $\boldsymbol{P}$ & $\boldsymbol{r}$ & $\boldsymbol{P}$ & $\boldsymbol{r}$ \\
\hline ER & - & - & 0.53 & 0.002 & -0.29 & 0.016 & -0.38 & 0.003 \\
\hline PR & 0.53 & 0.002 & - & - & -0.26 & 0.023 & -0.35 & 0.009 \\
\hline HER-2 & -0.29 & 0.016 & -0.26 & 0.023 & - & - & 0.61 & $<0.001$ \\
\hline Ki67 & -0.38 & 0.003 & -0.35 & 0.009 & 0.61 & $<0.001$ & - \\
\hline
\end{tabular}


Table 3 Relationship Between the Expression of ER, PR, HER-2 and Ki67 and the Characteristics of Ultrasound Signs in Breast Cancer Patients

\begin{tabular}{|c|c|c|c|c|c|c|c|c|}
\hline & $\operatorname{ER}(+)(n=7 I)$ & $P$ & PR (+) $(n=62)$ & $P$ & HER-2 (+) $(n=65)$ & $P$ & Ki-67 (+) $(n=86)$ & $P$ \\
\hline \multicolumn{9}{|l|}{ Tumor diameter $(\mathrm{cm})$} \\
\hline$<2$ & $33(64.71 \%)$ & 0.102 & $29(56.86 \%)$ & 0.137 & $24(47.06 \%)$ & 0.447 & $27(52.94 \%)$ & 0.004 \\
\hline$\geq 2$ & $38(50.00 \%)$ & & $33(43.42 \%)$ & & $4 I(53.95 \%)$ & & $59(77.63 \%)$ & \\
\hline \multicolumn{9}{|l|}{ Lymph node metastasis } \\
\hline Yes & $17(43.59 \%)$ & 0.003 & $13(33.33 \%)$ & 0.020 & $27(69.23 \%)$ & 0.007 & $32(82.05 \%)$ & 0.021 \\
\hline No & $64(72.73 \%)$ & & $49(55.68 \%)$ & & $38(43.18 \%)$ & & $54(61.36 \%)$ & \\
\hline \multicolumn{9}{|l|}{ Spicule sign } \\
\hline Yes & $39(69.64 \%)$ & 0.006 & $32(57.14 \%)$ & 0.096 & $25(44.64 \%)$ & 0.191 & $33(58.93 \%)$ & 0.060 \\
\hline No & $32(45.07 \%)$ & & $30(42.25 \%)$ & & $40(56.34 \%)$ & & $53(74.65 \%)$ & \\
\hline \multicolumn{9}{|l|}{ Calcifications } \\
\hline Yes & $30(63.83 \%)$ & 0.168 & $26(55.32 \%)$ & 0.261 & $26(55.32 \%)$ & 0.475 & $36(76.60 \%)$ & 0.101 \\
\hline No & $4 I(5 I .25 \%)$ & & $36(45.00 \%)$ & & $39(48.75 \%)$ & & $50(62.50 \%)$ & \\
\hline \multicolumn{9}{|l|}{ Blood flow grading } \\
\hline $0-1$ & $26(59.09 \%)$ & 0.598 & $19(43.18 \%)$ & 0.355 & $19(43.18 \%)$ & 0.189 & $24(54.55 \%)$ & 0.021 \\
\hline $2-3$ & $45(54.22 \%)$ & & $43(51.81 \%)$ & & $46(55.42 \%)$ & & $62(74.70 \%)$ & \\
\hline
\end{tabular}

LNM and Ki-67 high expression were risk factors for DFS (Table 4).

\section{Discussion}

Breast cancer consists of varied molecular subtypes since it has different molecular biological features and clinical behaviors. Sometimes, even varied molecular biological features and outcomes can be found in one of the breast cancer molecular subtypes. ${ }^{17}$ In addition, due to the invisible and not noticeable early symptoms of breast cancer, early screening is needed to retard deterioration of breast cancer to some extent. In this present research, we combined several indices (ER, PR, HER2 and Ki-67) for better judge the prognosis of breast cancer, and the corresponding conclusion suggested that the joint detection of multiple indicators is helpful for judging the prognosis of breast cancer.

Firstly, positive rate of ER, PR and HER-2 as well as Ki-67 high expression in breast cancer patients was elevated, and the positive rates of ER, PR and HER-2 were $73.36 \%$ (201/274), 59.85\% (164/274), and $24.09 \%$ (66/
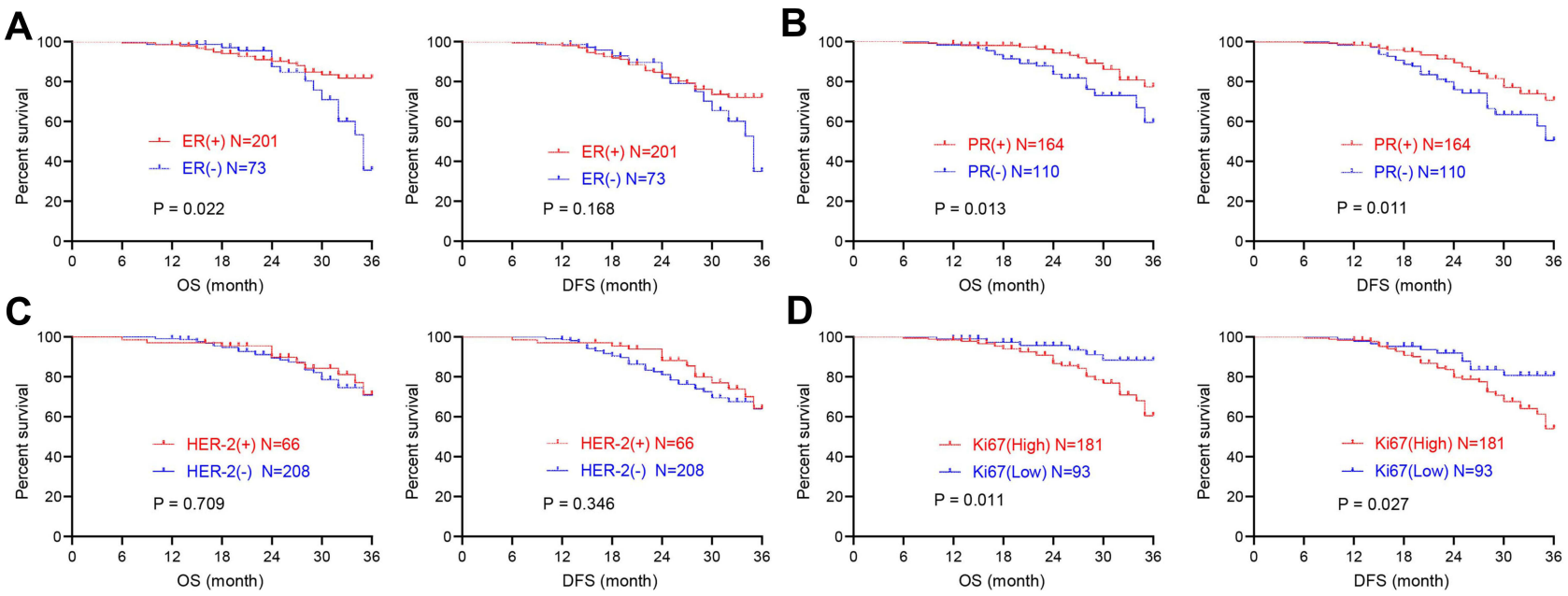

Figure 2 The relationship between ER, PR, HER-2 and Ki67 expression and prognosis in breast cancer patients. (A) Kaplan-Meier analysis for the correlation of ER-positive expression with OS and DFS. (B) Kaplan-Meier analysis for the correlation of PR-positive expression with OS and DFS. (C) Kaplan-Meier analysis for the correlation of HER-2-positive expression with OS and DFS. (D) Kaplan-Meier analysis for the correlation of Ki-67 high expression with OS and DFS.

Abbreviations: OS, overall survival; DFS, disease-free survival. 
Table 4 COX Multivariate Regression Analysis for Determining the Factors Affecting Overall Survival and Disease-Free Survival in Breast Cancer Patients

\begin{tabular}{|c|c|c|c|c|c|c|}
\hline \multirow[t]{2}{*}{ Characteristics } & \multicolumn{3}{|l|}{ OS } & \multicolumn{3}{|l|}{ DFS } \\
\hline & $\boldsymbol{P}$ & HR & $95 \% \mathrm{Cl}$ & $\boldsymbol{P}$ & HR & $95 \% \mathrm{Cl}$ \\
\hline TNM stage & 0.034 & 2.19 & $1.07-5.90$ & - & - & - \\
\hline Tumor diameter $(\mathrm{cm})$ & - & - & - & 0.044 & 2.56 & $1.02-7.16$ \\
\hline Lymph node metastasis & - & - & - & 0.025 & 1.83 & $1.06-5.34$ \\
\hline ER & 0.018 & 0.35 & $0.14-0.62$ & - & - & - \\
\hline $\mathrm{Ki}-67$ & 0.010 & 0.20 & $0.09-0.48$ & 0.021 & 0.23 & $0.11-0.57$ \\
\hline
\end{tabular}

Abbreviations: OS, overall survival; DFS, disease-free survival; $\mathrm{HR}$, hazard ratio; $\mathrm{Cl}$, confidence interval.

274); and high expression rate of Ki-67 was $66.06 \%$ (181/ 274), respectively. Breast cancer patients who express no ER are impossible to benefit from endocrine therapy, and ER status evaluation is therefore widely applied to breast cancers. ${ }^{18}$ PR, encoded by PGR, consists of 933 amino acids, and PR expression is regarded as a biomarker for normal ER functions in breast cancer. ${ }^{19}$ HER2 protein and ligand are revealed to participate in physiological activities of breast cancer cells for the purpose of inducing their proliferation, activation and differentiation. ${ }^{20}$ It is reported that tumor growth, invasion or metastasis related to HER2 overexpression in breast cancer. ${ }^{21}$ Since Ki67 is essential in cell proliferation, the value of which in assessing cell proliferation grade in breast cancer have been verified. ${ }^{22}$

Additionally, whether ER, PR, HER-2 and Ki-67 were correlation with each other in breast cancer patients were elevated, and we found a positive correlation between ER and PR, or HER-2 and Ki-67, a negative correlation between ER and HER-2, or ER and Ki-67, or PR and HER-2 or PR and Ki-67. Meanwhile, we next investigated the relationships between ER, PR, HER-2 and Ki-67 expression levels in breast cancer tissues and the characteristics of ultrasound signs in breast cancer patients. This research showed that ER-positive expression was related to LNM and blood flow grading; HER-2-positive expression was associated with LNM, while Ki-67 high expression was related to the tumor diameter, LNM, as well as blood flow grading. Consistent with our findings, HER2 expression has been revealed to positively correlates with tumor size, clinical TNM stage and LNM, while negatively correlates with ER/PR expression and tissue grade, implying that HER2 expression could be considered as a marker for breast cancer. ${ }^{7}$ Another study has indicated that Ki-67 expression has a positive correlation with TNM stage and LNM, and a negative correlation with tissue grade and ER/PR expression. ${ }^{7}$

Furthermore, we wanted to elucidate the relationships between ER, PR, HER-2 and Ki-67 expression and prognosis in breast cancer tissues. Kaplan-Meier analysis showed that PR protein-positive and Ki-67 high expression were related to OS and DFS. Further COX multivariate regression analysis suggested that LNM and Ki-67 high expression were risk factors for OS, and PR-positive was a protective factor for OS; TNM stage, tumor diameter, LNM and Ki-67 high expression were risk factors for DFS in breast cancer patients. In terms of the prognostic function, as previously described, ER and PR in breast cancer patients often exhibit better prognosis in contrast to those patients who lacking of these receptors. ${ }^{23}$ A prior article has revealed that Ki67 expression is regarded as a pivotal prognostic factor for both recurrence-free interval (RFI) and OS in ER-positive and HER2-negative breast cancer patients, and Ki67 expression remains essential for RFI and OS. ${ }^{24}$ Kleer et al have proposed that EZH2 overexpression has a close relationship with tumor size, ER/PR-negative status, advanced disease stage and declined DFS and OS in primary breast cancer. ${ }^{25}$ All these mentioned above further confirmed our findings.

To conclude, this research highlights that ER, PR, HER-2 and Ki-67 are independent and interrelated in breast cancer, which have a association with the ultrasound signs and prognosis of breast cancer. Through the joint detection of multiple indicators, the prognosis of breast cancer can be judged more accurately, which provides a reference for the individualized treatment of targeted drugs. Nevertheless, the limited sample 
size and the short follow-up time make it necessary to expand the sample size and extend the follow-up time to further analyze its observation in the long-term prognosis. Therefore, molecular diagnosis has become essential in diagnosis and therapy of breast cancer.

\section{Highlights}

1. ER, PR, HER-2 and Ki67 in breast cancer are both independent and interrelated.

2. ER, PR, HER-2 and Ki67 is related to different ultrasound signs of breast cancer.

3. PR-positive and Ki67 high expression are associated with OS and DFS of breast cancer.

4. Breast cancer prognosis is judged accurately with detection of multiple indicators.

5. This study offers a basis for individualized treatment of targeted drugs in breast cancer.

\section{Funding}

There is no funding to report.

\section{Disclosure}

The authors report no conflicts of interest in this work.

\section{References}

1. Yamamoto-Ibusuki M, Yamamoto Y, Yamamoto S, et al. Comparison of prognostic values between combined immunohistochemical score of estrogen receptor, progesterone receptor, human epidermal growth factor receptor 2, Ki-67 and the corresponding gene expression score in breast cancer. Mod Pathol. 2013;26(1):79-86. doi:10.1038/ modpathol.2012.151

2. Labrude P. The ephemeral historical museum of the Ecole Superieure de pharmacie de Nancy (1913-1937) Its collectionstoday. Rev Hist Pharm. 2014;62(381):47-60. doi:10.3406/pharm.2014.22696

3. Tu DG, Yu Y, Lee CH, et al. Hinokitiol inhibits vasculogenic mimicry activity of breast cancer stem/progenitor cells through proteasome-mediated degradation of epidermal growth factor receptor. Oncol Lett. 2016;11(4):2934-2940. doi:10.3892/ ol.2016.4300

4. Nie M, Qin Y, Zhu J, Li Y, Wang Z. Correlation between ultrasonic features and expression levels of C-erbB-2, VEGF and nm23 in breast cancer. Oncol Lett. 2018;16(2):1701-1707. doi:10.3892/ ol.2018.8777

5. Xu J, Guo X, Jing M, Sun T. Prediction of tumor mutation burden in breast cancer based on the expression of ER, PR, HER-2, and Ki-67. Onco Targets Ther. 2018;11:2269-2275. doi:10.2147/OTT.S159830

6. Tsai WC, Wei HK, Hung CF, et al. Better overall survival for breast cancer patients by adding breast ultrasound to follow-up examinations for early detection of locoregional recurrence-a Survival Impact Study. Ultrasound Med Biol. 2016;42(9):2058-2064. doi:10.1016/j. ultrasmedbio.2016.04.003

7. Song B, Wang L, Zhang Y, et al. Combined detection of HER2, Ki67, and GSTP1 genes on the diagnosis and prognosis of breast cancer. Cancer Biother Radiopharm. 2019;34(2):85-90. doi:10.1089/ cbr.2018.2570
8. Wang D, Zhu K, Tian J, et al. Clinicopathological and ultrasonic features of triple-negative breast cancers: a comparison with hormone receptor-positive/human epidermal growth factor receptor-2-negative breast cancers. Ultrasound Med Biol. 2018;44(5):1124-1132. doi:10.1016/j.ultrasmedbio.2018.01.013

9. Yan J, Liu XL, Han LZ, et al. Relation between Ki-67, ER, PR, Her2/neu, p21, EGFR, and TOP II-alpha expression in invasive ductal breast cancer patients and correlations with prognosis. Asian Pac J Cancer Prev. 2015;16(2):823-829. doi:10.7314/APJCP.2015.16.2.823

10. Hongisto V, Aure MR, Makela R, Sahlberg KK. The HER2 amplicon includes several genes required for the growth and survival of HER2 positive breast cancer cells - a data description. Genom Data. 2014;2:249-253. doi:10.1016/j.gdata.2014.06.025

11. Shilova ON, Proshkina GM, Lebedenko EN, Deyev SM. Internalization and recycling of the HER2 receptor on human breast adenocarcinoma cells treated with targeted phototoxic protein DARPinminiSOG. Acta Naturae. 2015;7(3):126-132. doi:10.32607/ 20758251-2015-7-3-126-132

12. Hao S, He ZX, Yu KD, Yang WT, Shao ZM. New insights into the prognostic value of $\mathrm{Ki}-67$ labeling index in patients with triple-negative breast cancer. Oncotarget. 2016;7(17):24824-24831. doi:10.18632/oncotarget.8531

13. Theriault RL, Carlson RW, Allred C, et al. Breast cancer, version 3.2013: featured updates to the NCCN guidelines. J Natl Compr Canc Netw. 2013;11(7):753-760; quiz 761. doi:10.6004/jnccn.2013.0098

14. Hammond ME, Hayes DF, Dowsett M, et al. American Society of Clinical Oncology/College of American Pathologists guideline recommendations for immunohistochemical testing of estrogen and progesterone receptors in breast cancer (unabridged version). Arch Pathol Lab Med. 2010;134(7):e48-e72. doi:10.5858/134.7.e48

15. Abdollahi A, Etemadi M. Pathological characteristics of triple-negative breast cancer at Main Referral Teaching Hospital, April 2014 to April 2015, Tehran, Iran. Int J Hematol Oncol Stem Cell Res. 2016;10(4):200-205.

16. Ahn S, Lee J, Cho MS, Park S, Sung SH. Evaluation of Ki-67 index in core needle biopsies and matched breast cancer surgical specimens. Arch Pathol Lab Med. 2018;142(3):364-368. doi:10.5858/arpa.2017-0014-OA

17. Wang XZ, Liu Q, Sun JJ, et al. Correlation between p53 and epidermal growth factor receptor expression in breast cancer classification. Genet Mol Res. 2015;14(2):4282-4290. doi:10.4238/2015.April.28.10

18. Ejlertsen B, Aldridge J, Nielsen KV, et al. Prognostic and predictive role of ESR1 status for postmenopausal patients with endocrineresponsive early breast cancer in the Danish cohort of the BIG 1-98 trial. Ann Oncol. 2012;23(5):1138-1144. doi:10.1093/annonc/ mdr438

19. Ding L, Zhang Z, Xu Y, Zhang Y. Comparative study of Her-2, p53, Ki-67 expression and clinicopathological characteristics of breast cancer in a cohort of northern China female patients. Bioengineered. 2017;8 (4):383-392. doi:10.1080/21655979.2016.1235101

20. Murthy P, Kidwell KM, Schott AF, et al. Clinical predictors of long-term survival in HER2-positive metastatic breast cancer. Breast Cancer Res Treat. 2016;155(3):589-595. doi:10.1007/ s10549-016-3705-3

21. Liu L, Chen F, Zhao J, Yu H. Correlation between overall survival and other endpoints in metastatic breast cancer with second- or third-line chemotherapy: literature-based analysis of 24 randomized trials. Bull Cancer. 2016;103(4):336-344. doi:10.1016/j. bulcan.2016.01.002

22. Shandiz FH, Shabahang H, Afzaljavan F, et al. Ki67 frequency in breast cancers without axillary lymph node involvement and its relation with disease-free survival. Asian Pac J Cancer Prev. 2016;17(3):1347-1350. doi:10.7314/APJCP.2016.17.3.1347

23. Brisken C. Reply to is progesterone a neutral or protective factor for breast cancer? Nat Rev Cancer. 2014;14(2):146. doi:10.1038/ $\operatorname{nrc3518-c2}$ 
24. Ohara M, Matsuura K, Akimoto E, et al. Prognostic value of Ki67 and p53 in patients with estrogen receptor-positive and human epidermal growth factor receptor 2-negative breast cancer: validation of the cut-off value of the Ki67 labeling index as a predictive factor. $\mathrm{Mol}$ Clin Oncol. 2016;4(4):648-654. doi:10.3892/mco.2016.776
25. Kleer CG, Cao Q, Varambally S, et al. EZH2 is a marker of aggressive breast cancer and promotes neoplastic transformation of breast epithelial cells. Proc Natl Acad Sci $U$ S A. 2003;100 (20):11606-11611. doi:10.1073/pnas.1933744100

\section{Publish your work in this journal}

Cancer Management and Research is an international, peer-reviewed open access journal focusing on cancer research and the optimal use of preventative and integrated treatment interventions to achieve improved outcomes, enhanced survival and quality of life for the cancer patient.
The manuscript management system is completely online and includes a very quick and fair peer-review system, which is all easy to use. Visit http://www.dovepress.com/testimonials.php to read real quotes from published authors. 